\title{
Optimising Ensemble of Two-Class classifiers using Spectral Analysis
}

\author{
T. Windeatt \\ Centre Vision Speech and Signal Processing (CVSSP) \\ University of Surrey, Guildford, Surrey, UK \\ t.windeatt@surrey.ac.uk
}

\begin{abstract}
An approach to approximating the decision boundary of an ensemble of two-class classifiers is proposed. Spectral coefficients are used to approximate the discrete probability density function of a Boolean Function. It is shown that the difference between first and third order coefficient approximation is a good indicator of optimal base classifier complexity. A theoretical analysis is supported by experimental results on a variety of Artificial and Real two-class problems.
\end{abstract}

Keywords-Boolean functions, ensemble classifier, multilayer perceptrons, pattern analysis, spectral analysis, supervised learning

\section{INTRODUCTION}

An important design issue for an ensemble of classifiers (Multiple Classifier System) is choice of individual (base) classifier complexity, which is usually determined with the help of a validation set or cross-validation techniques [1]. For solving a supervised learning problem, the maximum number of patterns should be reserved for training, which implies that base classifier parameters should ideally be determined from the training set. It may be possible to bootstrap training patterns and use the Ensemble Out-of-Bootstrap error estimate [2], in place of validation, but each bootstrap replicate uses approximately twothirds of the patterns, which may not be representative of the problem, for example for small sample size problems. In this paper, we only deal with the aggregation phase [16] of two class problems, but it should be possible to solve multi-class problems with this approach using Error-Correcting Output Coding (ECOC) [3].

Consider a simple ensemble framework of parallel base classifiers, each of which is given a binary decision, and if the problem is two-class, a Boolean mapping is defined between classifier decisions and target outputs. This mapping may be analysed using Walsh spectral coefficients, which was first proposed for Pattern Recognition in [4]. However, it was much later that first and second order Walsh spectral coefficients were used in the context of ensembles [5] [6]. The motivation for using Walsh coefficients in ensemble design is fully explored in [7]. For further understanding of the meaning and applications of Walsh coefficients see [8] and [9]. Since realistic learning problems are ill-posed [10], the Boolean function approximation will need to handle partially specified, noisy and possibly contradictory information.

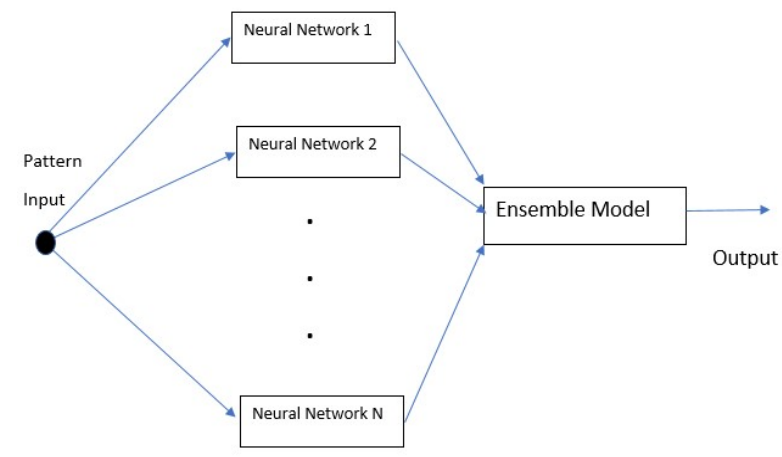

Fig. 1 Ensemble framework of parallel Neural Network Classifiers

\section{APPROXIMATING DISCRETE P.D.F.}

Consider the ensemble framework of Fig. 1, in which there are $N$ parallel Neural Network base classifiers, and let $X_{m}$ be the $N$-dimensional binary vector representing the $m t h$ training pattern, formed from the decisions of the $N$ classifiers. For a twoclass supervised learning problem of $\mu$ training patterns, the target label given to each pattern $X_{m}$ is denoted by $\Omega_{m}=\Phi\left(X_{m}\right)$ where $m=1 \ldots \mu$, and $\Phi$ is the unknown Boolean function that maps $X_{m}$ to $\Omega_{m}$. Therefore, the vector $X_{m}$ represents the $m t h$ original training pattern

$$
X_{m}=\left(x_{m 1}, x_{m 2}, \ldots, x_{m N}\right)
$$

where $X_{m}$ is a vertex in the $\mathrm{N}$-dimensional binary hypercube. Both pattern features and target label are binary, so that $x_{m i}, \Omega_{m} \in\{0,1\}$ or $\{1,-1\}$ where $i=1 \ldots N$. Using $\{1,-1\}$ coding, the Walsh transform of $\Phi$ is derived from the mapping $T_{n}$ and defined recursively as follows 


$$
T_{n}=\left[\begin{array}{cc}
T_{n-1} & T_{n-1} \\
T_{n-1} & -T_{n-1}
\end{array}\right], T_{1}=\left[\begin{array}{cc}
1 & 1 \\
1 & -1
\end{array}\right]
$$

The first, second and third order spectral coefficients derived from (1) are defined in [8] as follows

$$
\begin{gathered}
s_{i}=\frac{1}{2^{N} \mu} \sum_{m=1}^{\mu} x_{m i} \Omega_{m} \\
s_{i j}=\frac{1}{2^{N} \mu} \sum_{m=1}^{\mu}\left(x_{m i} \oplus x_{m j}\right) \Omega_{m} \\
s_{i j k}=\frac{1}{2^{N} \mu} \sum_{m=1}^{\mu}\left(x_{m i} \oplus x_{m j} \oplus x_{m k}\right) \Omega_{m}
\end{gathered}
$$

In (2) $s_{i}$ represents the correlation between $\Omega_{m}$ and $x_{i}$, and in (3) $s_{i j}$ represents correlation between $\Omega_{m}$ and $x_{m i} \oplus x_{m j}$, where $\oplus$ is logic Exclusive-OR. Third order coefficients (4) and higher follow the same pattern, but in this paper we restrict ourselves to first and third order spectral coefficients.

We now switch to $\{0,1\}$ coding to approximate the discrete probability density function (p.d.f.), $p\left(X=X_{j}\right)$ where $j$ can take any of $\mu$ possible values. From the p.d.f., we are able to calculate the probability of occurrence of the binary patterns. The following approach is similar to [4], but uses binary classifier space rather than binary patterns in the original feature space. A good choice of basis functions for this problem is the Rademacher-Walsh (RW) polynomials, which contain $2^{N}$ terms and are formed by taking products of distinct terms of the form $\left(2 x_{m i}-1\right)$. Table I shows the RW discrete polynomial functions $\varphi_{j}(X)$ which are orthogonal, satisfying the property that

$$
\sum_{m=1}^{2^{N}} \varphi_{j}\left(X_{m}\right) \varphi_{k}\left(X_{m}\right)= \begin{cases}2^{N} & \text { if } j=k \\ 0 & \text { if } j \neq k\end{cases}
$$

An approximation using $q$ basis functions and $\mu$ patterns is given by

$$
\hat{p}(X)=\sum_{j=1}^{q} c_{j} \varphi_{j}(X)
$$

where coefficients

$$
c_{j}=\frac{1}{2^{N} \mu} \sum_{i=1}^{\mu} \varphi_{j}\left(X_{i}\right)
$$

As an example of computing the functional approximation using basis functions, consider the linear approximation of the following non-separable Boolean function, taken from [6], ( see [6] for the computation of spectral coefficients in $\{1,-1\}$ coding). Table II shows the truth table in $\{0,1\}$ coding.
TABLE I. RADEMACHER-WALSH POLYNOMIALS

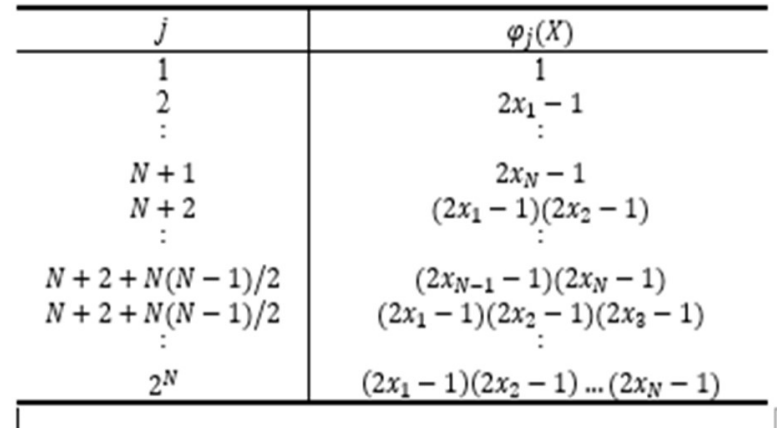

TABLE II. TRUTH TABLE BOOLEAN FUNCTION

\begin{tabular}{c|cccc}
$X_{m}$ & $X_{m 1}$ & $X_{m 2}$ & $X_{m 3}$ & $\Omega_{m}$ \\
\hline$X_{1}$ & 0 & 0 & 0 & 0 \\
$X_{2}$ & 1 & 0 & 0 & 1 \\
$X_{3}$ & 0 & 1 & 0 & 1 \\
$X_{4}$ & 1 & 1 & 0 & 0 \\
$X_{5}$ & 0 & 0 & 1 & 0 \\
$X_{6}$ & 1 & 0 & 1 & 1 \\
$X_{7}$ & 0 & 1 & 1 & 1 \\
$X_{8}$ & 1 & 1 & 1 & 1 \\
\hline
\end{tabular}

A single decision function will be formed by subtracting individual decision functions for the two classes using the linear approximation basis functions from (6) and (7). The three class $\omega_{0}$ patterns are given by $(0,0,0),(1,1,0),(0,0,1)$ and five class $\omega_{1}$ patterns by $(1,0,0),(0,1,0),(1,0,1),(0,1,1),(1,1,1)$. From Table I, the linear approximation uses four basis functions denoted by

$$
\begin{array}{cc}
\varphi_{1}(X)=1, & \varphi_{2}(X)=\left(2 x_{1}-1\right), \\
\varphi_{4}(X)=\left(2 x_{3}-1\right) & \varphi_{3}(X)=\left(2 x_{2}-1\right),
\end{array}
$$

The factor $\frac{1}{2^{N}}$ is common to all terms in (7), so can be neglected in the computation. For class $\omega_{1}$ the coefficients are given by

$$
\begin{aligned}
& c_{1}=\frac{1}{5} \sum_{i=1}^{5} \varphi_{1}\left(X_{i}\right)=\frac{1}{5}(+1+1+1+1+1)=1 \\
& c_{2}=\frac{1}{5} \sum_{i=1}^{5} \varphi_{2}\left(X_{i}\right)=\frac{1}{5}(+1-1+1-1+1)=\frac{1}{5} \\
& c_{3}=\frac{1}{5} \sum_{i=1}^{5} \varphi_{3}\left(X_{i}\right)=\frac{1}{5}(-1+1-1+1+1)=\frac{1}{5} \\
& c_{4}=\frac{1}{5} \sum_{i=1}^{5} \varphi_{4}\left(X_{i}\right)=\frac{1}{5}(-1-1+1+1+1)=\frac{1}{5}
\end{aligned}
$$

The density function linear approximation for class $\omega_{1}$ 


$$
\hat{p}\left(X \mid \omega_{1}\right)=1+\frac{1}{5}\left(2 x_{1}-1\right)+\frac{1}{5}\left(2 x_{2}-1\right)+\frac{1}{5}\left(2 x_{3}-1\right)
$$

Similar analysis for class $\omega_{0}$ gives

$$
\hat{p}\left(X \mid \omega_{0}\right)=1-\frac{1}{3}\left(2 x_{1}-1\right)-\frac{1}{3}\left(2 x_{2}-1\right)-\frac{1}{3}\left(2 x_{3}-1\right)
$$

Assuming prior probabilities can be determined from the number of patterns, so $p\left(\omega_{1}\right)=\frac{5}{8}, p\left(\omega_{0}\right)=\frac{3}{8}$, the decision functions are

$$
\begin{aligned}
\hat{p}\left(X \mid \omega_{1}\right) p\left(\omega_{1}\right)= & \frac{5}{8}+\frac{1}{8}\left(2 x_{1}-1\right)+\frac{1}{8}\left(2 x_{2}-1\right) \\
& +\frac{1}{8}\left(2 x_{3}-1\right) \\
\hat{p}\left(X \mid \omega_{0}\right) p\left(\omega_{0}\right)= & \frac{3}{8}-\frac{1}{8}\left(2 x_{1}-1\right)-\frac{1}{8}\left(2 x_{2}-1\right) \\
& -\frac{1}{8}\left(2 x_{3}-1\right)
\end{aligned}
$$

Subtracting the decision functions gives combined decision function

$$
d(X)=\frac{1}{4}\left(1+\left(2 x_{1}-1\right)+\left(2 x_{2}-1\right)+\left(2 x_{3}-1\right)\right)
$$

It is easy to verify that the decision function $d(X)$ in (8), based on the linear approximation, does not separate the patterns of the non-separable function, as expected. Using Table II, note that $d(X)>0$ for patterns $X_{4}, X_{6}, X_{7}, X_{8}$ and $d(X)<0$ for $X_{1}$, but all other patterns have $d(X)=0$.

It is possible to determine the coefficients directly by counting the correlation with the class label, as given in (2). In the above example, note that if class label agrees with $x_{i}$ then add +1 , otherwise add -1 . For example, the first order coefficient in (8) $s_{1}$ is given by

$s_{1}=\frac{1}{\mu} \sum_{m=1}^{\mu} \mathcal{C}\left(x_{m 1}, \Omega_{m}\right) \quad$ where $\mathcal{C}(a, b)=\left\{\begin{array}{l}+1 \text { if } a=b \\ -1 \text { if } a \neq b\end{array}\right.$

Therefore $\quad s_{1}=\frac{1}{8}(1+1-1-1+1+1-1+1)=\frac{2}{8}$

For third order coefficient, neglecting the factor $\frac{1}{2^{N}}$

$$
s_{123}=\frac{1}{\mu} \sum_{m=1}^{\mu} \mathcal{C}\left(x_{m 1} \oplus x_{m 2} \oplus x_{m 3}, \Omega_{m}\right)
$$

If we now assume that there are only three class $\omega_{1}$ patterns with $X_{2}, X_{3}$ missing and two class $\omega_{0}$ patterns with $X_{4}$ missing, we can repeat the calculation for the combined decision function with $X_{1}, X_{5}, X_{6}, X_{7}, X_{8}$ in Table II giving

$$
d(X)=\frac{1}{5}\left(1+3\left(2 x_{1}-1\right)+3\left(2 x_{2}-1\right)+3\left(2 x_{3}-1\right)\right)
$$

which perfectly separates the five training patterns.

\section{TUMER-GHOSH MODEL}

Fig. 2 shows the two class $\left(\omega_{1}, \omega_{0}\right)$ model of Added Classification Error according to [11], which for simplicity is restricted to one dimension $(x)$. The model assumptions are that the a posteriori probability distributions are approximated by

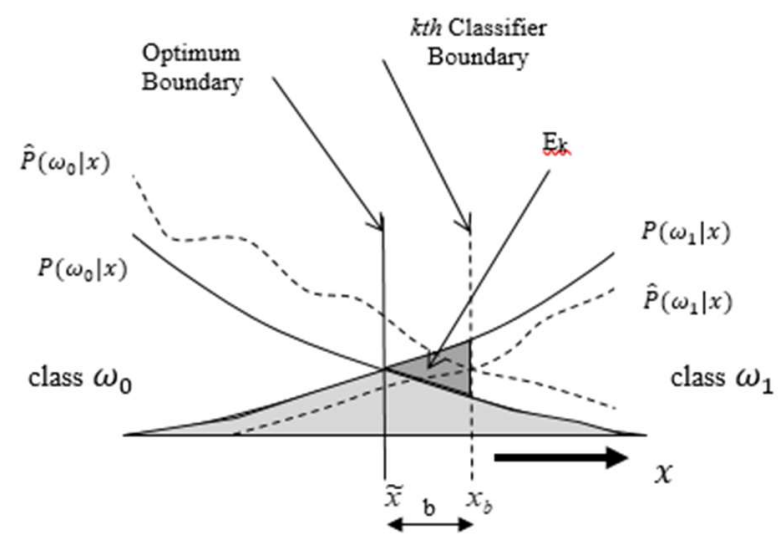

Fig. 2 Tumer-Ghosh model showing real and estimated probabilities and Added Error $E_{k}$ for $k$ th classifier shown as darkly shaded region

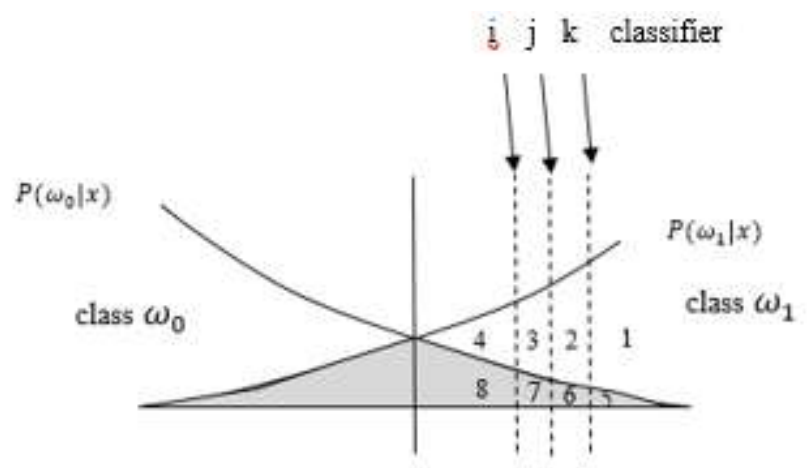

Fig. 3 Tumer Ghosh model showing $i, j, k t h$ classifier boundaries and areas 1-8

base classifier outputs and are locally monotonic around the Bayes boundary. While a Gaussian Distribution satisfies these properties, it is not necessary to assume overlapping Gaussians in the Tumer-Ghosh model [12].

The optimum (Bayes) boundary in Fig. 2 is the loci of all points $\tilde{x}: P\left(\omega_{1} \mid \tilde{x}\right)=P\left(\omega_{0} \mid \tilde{x}\right)$ and the output of the classifier representing class $\omega_{1}$ is given by

$$
\hat{P}\left(\omega_{1} \mid x\right)=P\left(\omega_{1} \mid x\right)+\varepsilon_{1}(x)
$$

where $P, \hat{P}$ are the actual and estimated a posteriori probability distributions as shown in Fig. 2, and $\varepsilon_{1}(x)$ is the difference between them. A similar equation to (9) is obtained for class $\omega_{0}$ with $P\left(\omega_{0} \mid x\right), \hat{P}\left(\omega_{0} \mid x\right)$ and error $\varepsilon_{0}(x)$. If $b$ in Fig. 2 is the amount that the $k$ th classifier boundary $\left(\mathrm{x}_{\mathrm{b}}\right)$ differs from the ideal Bayes boundary ( $\widetilde{x})$, and assuming that $b$ is a Gaussian random variable with mean $\beta$ and variance $\sigma_{\mathrm{b}}$, in [11] it is shown using (9) that Added Classification Error for $k$ th classifier is given by 


$$
E_{k}=\nabla P\left(\sigma_{b}^{2}+\beta^{2}\right)
$$

$$
\text { where } \nabla P=0.5\left(P^{\prime}\left(\omega_{1} \mid \tilde{x}\right)-P^{\prime}\left(\omega_{0} \mid \tilde{x}\right)\right) p(\tilde{x}) \text { and } P^{\prime}
$$

indicates differentiation.

In this paper, the model is extended to the case of a triple of classifiers, and we assume in the analysis that classifier complexity is varied from under to over-fitting, with respect to optimal. Fig. 3 shows decision boundaries of $(i, j . k)$ th classifiers for which it is assumed that the complexity is not sufficient to approximate the Bayes boundary, so that all classifiers under-fit. Note in Fig. 3 that estimated probabilities $\hat{P}\left(\omega_{0} \mid x\right)$ and $\hat{P}\left(\omega_{1} \mid x\right)$ are omitted for clarity. Mutually exclusive areas under the probability distribution are labelled $1-8$ in Fig. 3 and area y is given by $\mathrm{a}_{\mathrm{y}} . E_{i}$ corresponds to $\mathrm{a}_{4}, E_{j}$ to $\mathrm{a}_{4}+\mathrm{a}_{3}, E_{k}$ to $\mathrm{a}_{4}+\mathrm{a}_{3}+\mathrm{a}_{2}$.

We define $n_{p q}$ to be the number of class $\omega_{p}$ patterns for which $\varphi$ has value $q$ where $p, q \in\{0,1\}$. Table III shows the first order contribution for each area for the $k t h$ classifier. For example, $a_{1}$ and $a_{5}$ show $n_{11}$ for class $\omega_{1}$ since patterns are correctly classified by the $k t h$ classifier. Assuming that there are approximately equal number of class $\omega_{1}$ and class $\omega_{0}$ patterns under the tail of the distribution in areas $a_{5}$ to $a_{8}$, the contributions cancel, so that we need only consider $\mathrm{a}_{1}$ to $\mathrm{a}_{4}$. All three classifiers correctly classify class $\omega_{0}$ patterns, except in areas $a_{5}, a_{6}, a_{7}$ so we can ignore class $\omega_{0}$ patterns in the analysis. Table IV shows the first order contributions for the three classifiers individually, and the last row $i j k$ corresponds to third order contribution. For example, for $a_{1}$ the individual $\varphi\left(x_{i}\right)$ for each classifier is 1 so third order $\varphi\left(x_{i} \oplus x_{j} \oplus x_{k}\right)=$ $1 \oplus 1 \oplus 1=1$. Positive correlation is indicated by $n_{11}$ and negative correlation by $n_{10}$.

For the first order, we see that the coefficients corresponding to $k, j, i$ th classifier are respectively $\left(a_{1}-a_{2}-a_{3}-a_{4}\right),\left(a_{1}+\right.$ $\left.a_{2}-a_{3}-a_{4}\right)$ and $\left(a_{1}+a_{2}+a_{3}-a_{4}\right)$. Therefore, the $i$ th classifier carries more weight than the $j$ th classifier, which carries more weight than the $k t h$ classifier. The result is that the decision boundary for $W 1$ would be closer to optimal, compared to $M V$ which for the three classifiers would coincide with the $j t h$ classifier boundary. The third order coefficient is given by $a_{1}-$ $\left.a_{2}+a_{3}-a_{4}\right)$. By inspection of Fig. 3 , we can see that $a_{2}+a_{4}$ is likely to be less than $a_{1}+a_{3}$, so we expect an overall positive correlation, which when added to the first order contribution would move the decision boundary closer to $\tilde{x}$.

Now consider the case that base classifiers are optimal, and assume that classifier $j$ is close to $\tilde{x}$, and classifiers $i, k$ are either side of the Bayes boundary. A similar analysis shows that the ensemble boundary for $W 1$ would then be close to $\tilde{x}$. Since the areas are approximately equal, the third order contribution adds little to the first order. We may also expect that the Boolean function would be quite complex, with approximately equal number of patterns in the two classes under the tail. It may be seen in Section IV that $W 3$ training error is sometimes higher than $W 1$ training error, when base classifier is optimised.
TABLE III. FIRST ORDER CONTRIBUTIONS FOR KTH CLASSIFIER IN FIG. 3

\begin{tabular}{l|cccccccc}
\hline & $a_{1}$ & $a_{2}$ & $a_{3}$ & $a_{4}$ & $a_{5}$ & $a_{6}$ & $a_{7}$ & $a_{8}$ \\
\hline$\omega_{1}$ & $n_{11}$ & $n_{10}$ & $n_{10}$ & $n_{10}$ & $n_{11}$ & $n_{10}$ & $n_{10}$ & $n_{10}$ \\
\hline$\omega_{0}$ & & & & & $n_{01}$ & $n_{00}$ & $n_{00}$ & $n_{00}$ \\
\hline
\end{tabular}

TABLE IV. FIRST AND THIRD ORDER CONTRIBUTIONS FOR I,J,K TH CLASSIFIERS IN FIG. 3 , FOR CLASS $\omega_{1}$

\begin{tabular}{c|cccc}
\hline & $a_{1}$ & $a_{2}$ & $a_{3}$ & $a_{4}$ \\
\hline $\mathrm{k}$ & $\mathrm{n}_{11}$ & $\mathrm{n}_{10}$ & $\mathrm{n}_{10}$ & $\mathrm{n}_{10}$ \\
\hline $\mathrm{j}$ & $\mathrm{n}_{11}$ & $\mathrm{n}_{11}$ & $\mathrm{n}_{10}$ & $\mathrm{n}_{10}$ \\
\hline $\mathrm{i}$ & $\mathrm{n}_{11}$ & $\mathrm{n}_{11}$ & $\mathrm{n}_{11}$ & $\mathrm{n}_{10}$ \\
\hline $\mathrm{ijk}$ & $\mathrm{n}_{11}$ & $\mathrm{n}_{10}$ & $\mathrm{n}_{11}$ & $\mathrm{n}_{10}$ \\
\hline
\end{tabular}

TABLE V. DATASETS SHOWING NUMBER OF PATTERNS, NUMBER OF FEATURES AND OPTIMAL ESTIMATE

\begin{tabular}{l|l|lll}
\hline Data & Problem & \#pat & \#feat & $\% O E$ \\
\hline Real2 & card & 690 & 51 & 9.9 \\
Real2 & credita & 690 & 14 & 11.1 \\
Real2 & diabetes & 768 & 8 & 20.1 \\
Real2 & heart & 920 & 35 & 11.5 \\
Real2 & ion & 351 & 34 & 5.2 \\
Real2 & vote & 435 & 16 & 2.2 \\
Art2 & threenorm & 3000 & 20 & 10.5 \\
Art2 & highleym & 3000 & 2 & 5.1 \\
Art2 & circular & 3000 & 2 & 12.8 \\
Art2 & gaussimp & 3000 & 2 & 12.0 \\
Art2 & banana & 3000 & 2 & 1.2 \\
Art2 & lithuani & 3000 & 2 & 1.8 \\
\hline
\end{tabular}

\section{EXPERIMENTAL EVIDENCE}

There are two types of two-class problems labelled Real2, Art2 in Table $\mathrm{V}$ which shows the number of patterns, number of features and optimal error rate (OE), obtained by manually optimizing neural network base classifiers using 90/10\% train/test split. Real2 is selected from [13], and Art2 is artificial data taken from [14]. For the experiments, the random train/test split is $80 / 20$ for problems in Real2, and for Art2 there are 600 training patterns and 2400 testing patterns. Experiments for all datasets are repeated ten times and averaged. Test error rates will have OE subtracted, to give a fairer result when the average over different datasets is reported, so accuracy of $\mathrm{OE}$ is not crucial.

Neural networks (NN) are used as base classifiers as shown in Fig. 1. Each NN has the same architecture, a single hidden layer multi-layer perceptron with the same number of nodes and training epochs, using the Levenberg-Marquardt training algorithm with default parameters. The ensemble has 15 MLP base classifiers, the diversity in each being due to random starting weights. 
The nomenclature for test error rate is as follows, with training error rate indicated by adding $t r$.

$M V$ Majority vote combiner

$W 1, W 3$ Walsh coefficient combiner of order 1,3

$B A$ Base Classifier

$O E$ Optimal Estimate

Note that for computing $W 1, W 3$ as explained in Section II, there are no parameters to set, as with $M V$. The experiments are designed to test the hypothesis that the difference between W3tr and W1tr is a good indicator of optimal base classifier complexity.

Fig. 4 shows various plots for Credita dataset, combining NN classifiers that are systematically varied 1-69 training epochs (log scale) and 32 nodes. Fig. 4 (a) (b) show test error rates for $W 1$ and $B A$ with $O E$ subtracted. For the Credita dataset, the optimal value is at 4 epochs for $W 1$ and at 7 epochs for $B A$. As training epochs is increased the optimal value for $W 1$ may be lower than for $B A$, which is due to the accuracy/diversity tradeoff in ensemble classifiers [15]. Fig. 4 (c) shows the difference between W3tr and W1tr, and shows a peak at 4 epochs. Fig. 4 (d) shows the difference between $M V$ and $W 1$ test error rates, and note that $W 1$ is never worse than $M V$, and at low (1-2) epochs is superior. Note also at 4 epochs $(W 3-W 1) t r$ is positive so that W3tr is higher than W1tr, as explained in Section III.

Fig. 5 and 6 show the mean curves over Real2 and Art2 datasets, as training epochs is varied 1-69 and nodes 16,32. A similar trend to the Credita dataset may be observed, with ( W3tr - W1tr) predicting the optimal test error rate, at 3-4 epochs in Fig. 5 and at 4-7 epochs in Fig. 6. From Fig. 5 (d) and Fig. 6 (d) $W 1$ is to be preferred to $M V$ at 1-2 epochs and achieves similar to $M V$ for higher epochs.

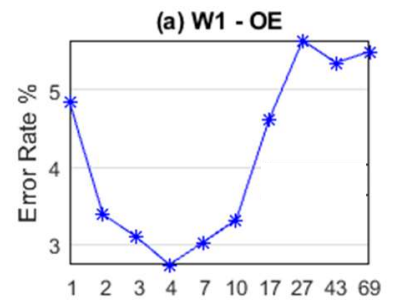

(c) $(\mathrm{W} 3-\mathrm{W} 1) \operatorname{tr}$

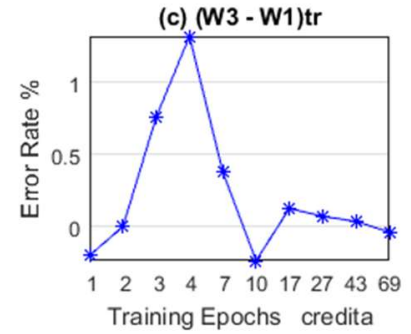

(b) BA - OE

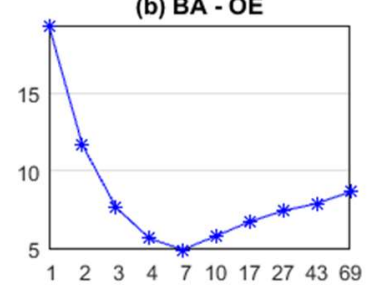

(d) MV - W1

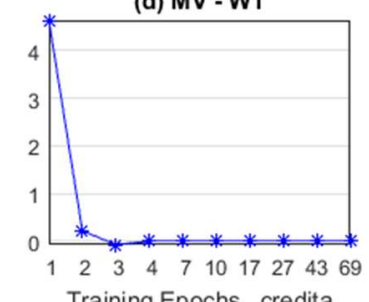

Training Epochs credita
Fig. 4. Credita data with NN 32 nodes and epochs varying 1-69 (a) W1 - OE

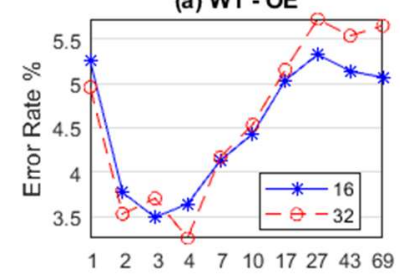

(c) $(\mathrm{W} 3-\mathrm{W} 1) \mathrm{tr}$

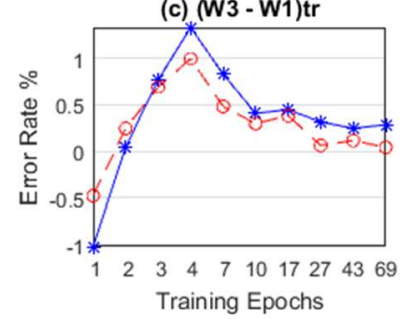

Fig. 5. Mean over six Real2 datasets with NN (a) W1 - OE

(c) (W3 - W1)tr
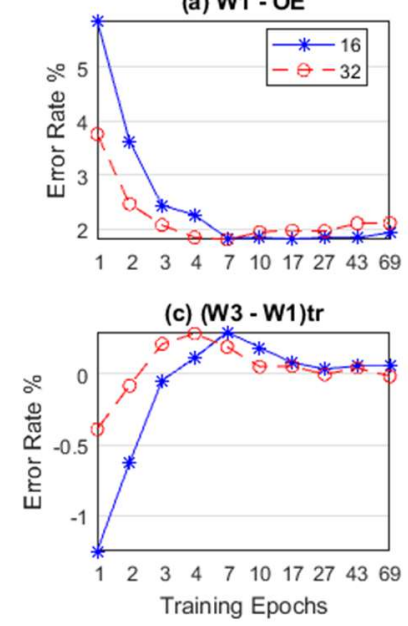

(b) BA - OE

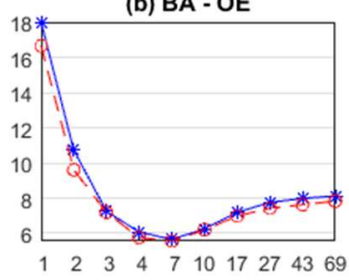

(d) MV - W1

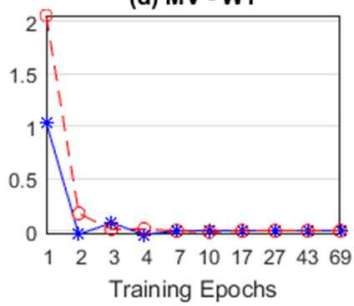

\section{6,32 nodes and epochs varying 1-69}

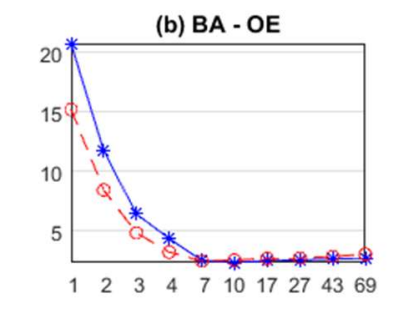

(d) MV - W1

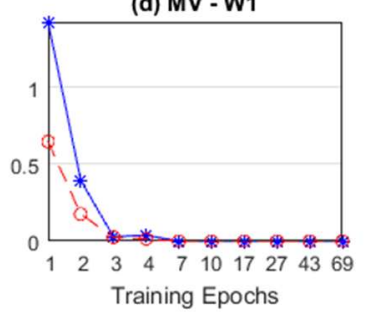

Fig. 6. Mean over six Art2 datasets with NN

16,32 nodes and epochs varying 1-69

To demonstrate how $W 3$ performs compared to $W 1$ and $M V$, example decision boundaries are shown for artificial data with two features. Ensemble decision boundaries for Highleyman dataset with 16 node NN base classifiers are shown for 10 epochs in Fig. 7, and for 2 epochs in Fig. 8. It can be seen from Fig. 7 that all ensemble decision boundaries achieve optimal performance at 10 epochs. Individual boundaries for seven base classifiers for 2 epochs are shown in Fig. 9, from which it may be seen that there is great variation in individual boundaries, but from Fig. 8, W3 at 2 epochs is closer to optimal than $W 1$ or $M V$. 


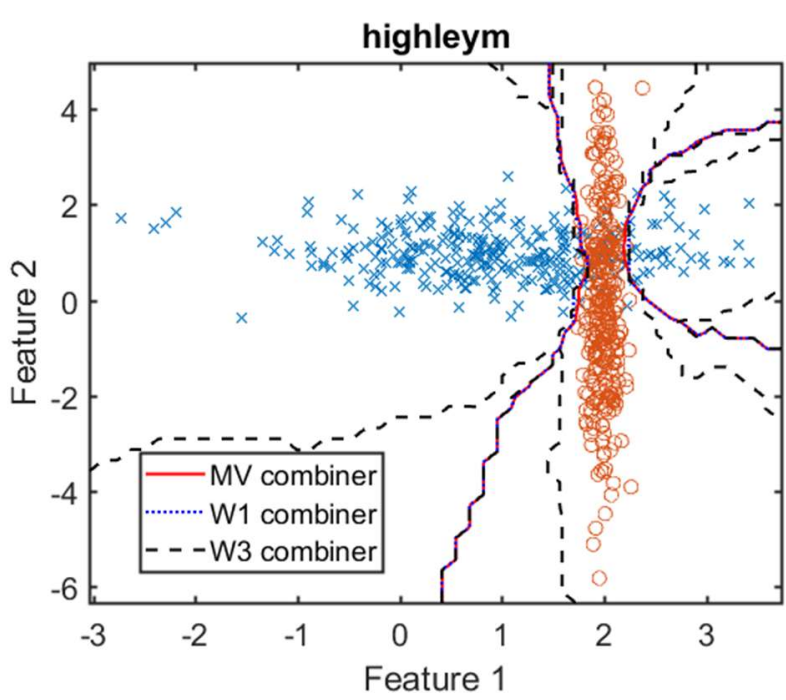

Fig. 7. Combiner boundaries Highleyman data 16 nodes and 10 epochs

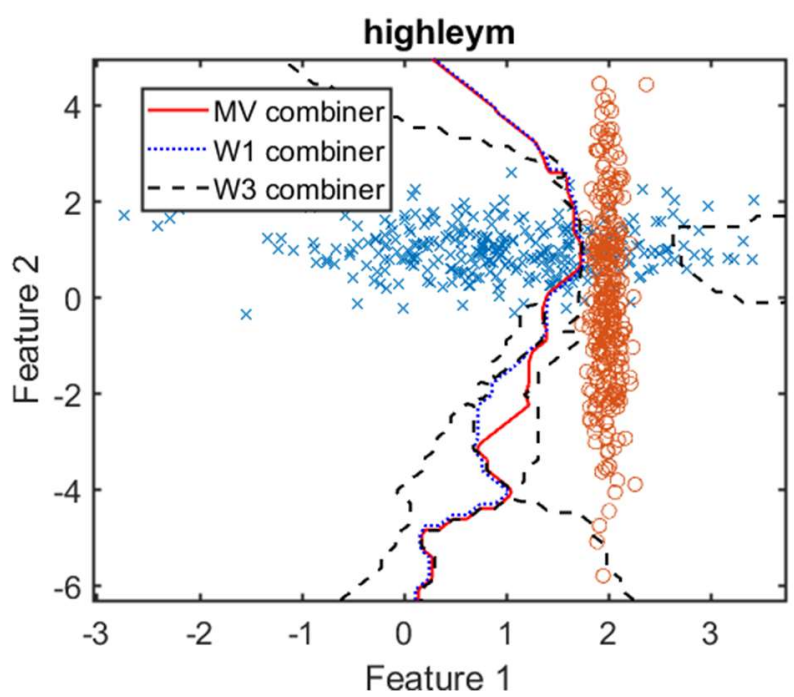

Fig. 8. Combiner boundaries Highleyman data 16 nodes and 2 epochs

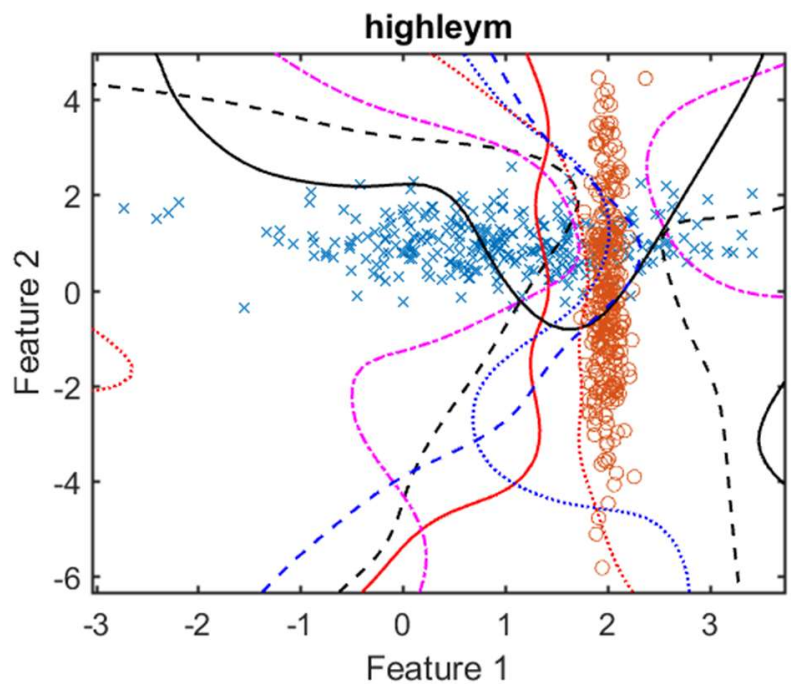

Fig. 9. Seven individual boundaries for Fig. 8

\section{CONCLUSION}

If a decision is taken for each base classifier in an ensemble that solves a supervised two-class learning problem, a Boolean function is defined. The function may be noisy, contradictory and ill-defined, so that a spectral analysis provides a useful approximation. In this paper, it is shown that the difference between third and first order Walsh approximation is a good indicator of optimal number of training epochs for a Neural Network classifier. Further work is aimed at using a spectral analysis to optimize other base classifier parameters, and to solve multi-class problems using the Error-Correcting Output Coding approach.

\section{REFERENCES}

[1] L. K. Hansen and P. Salamon, "Neural Network Ensembles, “ IEEE Trans. PAMI, vol. 12, pp. 993-1001, Oct. 1990.

[2] T. Bylander, "Estimating generalisation error two-class datasets using out-of-bag estimate," Machine Learning vol. 48, no. 1-3, pp. 287-297, 2002 .

[3] T. G. Dietterich and G. Bakiri, Solving Multi-class Learning Problems via Error-Correcting Output Codes, J. Artificial Intelligence Research vol. 2, 1995, 263-286.

[4] J.T Tou and R. C. Gonzales, Pattern Recognition Principles, AddisonWesley, 1974, pp. 151-4.

[5] T. Windeatt and C. Zor, "Minimising Added Classification Error using Walsh Coefficients", IEEE Trans Neural Networks, vol. 22 no. 8, pp. 1334- 1339, 2011

[6] T. Windeatt, C. Zor "Ensemble Pruning using spectral coefficients," IEEE Trans Neural Networks and Learning Systems, vol. 24, no. 4, pp. 673 $678,2013$.

[7] T. Windeatt, "Vote Counting Measures for Ensemble Classifiers," Pattern Recognition, vol. 36, no. 12, pp. 2743-2756, 2003.

[8] L. Hurst, D. M. Miller, and J. Muzio, Spectral Techniques in Digital Logic, Academic Press, 1985.

[9] K. G. Beauchamp, Walsh Functions and their Applications, Academic Press, 1975.

[10] A. N. Tikhonov and V. A. Arsenin, Solutions of Ill-posed Problems, Winston \& Sons, Washington, 1977.

[11] K. Tumer and J. Ghosh, "Error correlation and error reduction in ensemble classifiers," Connection Science, vol. 8, no. 3, pp. 385-404, 1996.

[12] L.I. Kuncheva, Combining Pattern Classifiers, Wiley, 2004.

[13] C. J. Merz and P. M. Murphy, UCI repository of ML databases, [Online], http://www.ics.uci.edu/ mlearn/MLRepository.html.

[14] R.P.W. Duin, P. Juszczak, P. Paclik, E. Pekalska, D. de Ridder, D.M.J. Tax, S. Verzakov PRTools4.1, A Matlab Toolbox for Pattern Recognition, Delft University of Technology, 2007.

[15] T. Windeatt, "Accuracy/ Diversity and ensemble classifier design," IEEE Trans Neural Networks, vol. 17, pp. 1194- 1211, Sept. 2006.

[16] R. M. O. Cruz, R. Sabourin and G. D. C. Cavalcanti, Dynamic classifier selection: Recent advances and perspectives, Information Fusion vol. 41, pp 195-216, 2018. 